\title{
Practitioner perspectives on building capacity for evidence-based public health in state health departments in the United States: a qualitative case study
}

Stephanie Mazzucca ${ }^{1 *}$ (D), Cheryl A. Valko ${ }^{1}$, Amy A. Eyler ${ }^{1}$, Marti Macchi², Andrew Lau ${ }^{3}$, Jeanne Alongi², John Robitscher ${ }^{2}$ and Ross C. Brownson ${ }^{1,4}$

\begin{abstract}
Background: Public health agencies are responsible for implementing effective, evidence-based public health programs and policies to reduce the burden of chronic diseases. Evidence-based public health can be facilitated by modifiable administrative evidence-based practices (A-EBPs) (e.g., workforce development, organizational climate), yet little is known about how practitioners view A-EBPs. Thus, the purpose of this qualitative study was to understand state health department practitioners' perceptions about how A-EBPs are implemented and what facilitators and barriers exist to using A-EBPs.

Methods: Chronic disease prevention and health promotion program staff who were members of the National Association of Chronic Disease Directors were recruited to participate in telephone interviews using a snowball sampling technique. Interviews were transcribed verbatim, and transcripts were analyzed using a common codebook and the a priori method in NVivo.

Results: Twenty seven interviews were conducted with practitioners in four states (5-8 interviews per state). All practitioners felt that their work unit culture is positive and that leadership encouraged and expected staff to use evidence-based processes. Participants discussed the provision of trainings and technical assistance as key to workforce development and how leaders communicate their expectations. Access to evidence, partnerships, and funding restrictions were the most commonly discussed barriers to the use of A-EBPs and EBDM.

Conclusions: Results of this study highlight practitioners' perspectives on promoting evidence-based public health in their departments. Findings can inform the development and refinement of resources to improve A-EBP use and organizational and leadership capacity of state health departments.
\end{abstract}

Keywords: Evidence-based practice, Public health practice, Workforce development, Chronic disease prevention

\footnotetext{
*Correspondence: smazzucca@wustl.edu

${ }^{1}$ Prevention Research Center in St. Louis, Brown School, Washington

University in St. Louis, One Brookings Drive, Campus Box 1196, St. Louis, MO

63130, USA

Full list of author information is available at the end of the article
}

(c) The Author(s). 2020 Open Access This article is distributed under the terms of the Creative Commons Attribution 4.0 International License (http://creativecommons.org/licenses/by/4.0/), which permits unrestricted use, distribution, and reproduction in any medium, provided you give appropriate credit to the original author(s) and the source, provide a link to the Creative Commons license, and indicate if changes were made. The Creative Commons Public Domain Dedication waiver (http://creativecommons.org/publicdomain/zero/1.0/) applies to the data made available in this article, unless otherwise stated. 


\section{Contributions to the literature}

- Implementing evidence-based public health is challenging but can be facilitated at the organizational level using administrative evidence-based practices (A-EBPs).

- In interviews with state health department chronic disease staff, practitioners reported successes (e.g., leaders dedicated to evidence-based public health) and challenges (e.g., forming and maintaining partnerships) to using A-EBPs.

- Knowledge of practitioners' views of how A-EBPs operate within their SHD can inform development of effective strategies to improve agency capacity that are relevant to practitioners and ultimately improve public health practice.

\section{Background}

The prevalence of and costs associated with chronic disease, including cardiovascular disease, cancer, and diabetes, across the globe are staggering, with an estimated $71 \%$ of deaths worldwide due to chronic disease [1]. Recent data suggest that $88 \%$ of deaths in 2016 in the United States (US) are attributable to chronic diseases [1], and chronic diseases account for $90 \%$ of the $\$ 3.5$ trillion spent annually on health care [2, 3]. Promotion of health behaviors including healthy eating, physical activity, avoiding tobacco, and participating in cancer screenings can prevent chronic diseases and manage complications of those with chronic diseases [4-8]. Thus, public health systems across the world have been tasked with intervening at the population level to reduce the burden of chronic disease, especially for groups of people experiencing inequities in health due to social determinants of health, such as insurance status, income, education, and the characteristics of environments in which people live (e.g., built environment) [9]. Additionally, many governmental agencies across the world have emphasized the need for public health systems to use evidence-based approaches in their chronic disease prevention efforts [10-13].

Evidence-based public health (EBPH) is an approach to public health practice in which public health practitioners identify, implement, and evaluate evidence-based interventions (EBIs), including those focused on chronic disease prevention. EBPH is characterized by the use of evidence-based decision-making (EBDM), which is the process of integrating the best available research evidence, practitioner expertise, and the characteristics, needs, and preferences of the community $[14,15]$. EBDM allows public health practitioners to identify, implement, and evaluate evidence-based programs and policies that are relevant for their communities [14]. There is an ethical justification for using of EBDM [16, 17], and EBDM is associated with the improved use of evidence-based interventions (EBIs) and public health agency performance [18-22]. Additionally, EBDM can facilitate the efficient use of finite resources, as this process helps prioritize implementation of programs and policies that can have the biggest impact on the public's health given the financial and personnel investments of a public health program (i.e., maximizing costeffectiveness). Practitioners and researchers have emphasized the use of EBDM to improve public health practice [23-28], and EBDM components are prominent in US Public Health Accreditation Board standards [29]. Therefore, there is a need for efforts to better understand and foster EBDM within public health agencies.

For EBDM to operate efficiently, individual practitioners must have appropriate, relevant skills and the organizations in which they work must have sufficient resources, infrastructure, and leadership [30]. Administrative evidencebased practices (A-EBPs) are structures and activities at the organizational level that can facilitate evidence-based decision-making in health departments, including practices such as provision of skills-based training, leaders setting expectations for EBDM in strategic plans, and allocating financial resources for quality improvement and implementation of programs [23, 31]. A-EBPs can be grouped into five domains: workforce development, organizational climate and culture, leadership, relationships and partnerships, and financial processes. A-EBPs can be positively associated with performance measures, e.g., achieving core public health functions, carrying out evidence-based interventions [32], can potentially be modified over the course of a few years, and are within the control of public health agencies. Thus, understanding how A-EBPs operate within public health agencies and what is needed to support them can inform strategies to build capacity for EBDM within public health agencies.

Three levels of government operate in the United States to conduct public health: national, state, and local. At the national level, the Department of Health and $\mathrm{Hu}$ man Services (DHHS) Agencies with the DHHS, e.g., the Centers for Disease Control and Prevention (CDC), establish funding priorities and distribute public funds to state and local health departments for specific chronic disease prevention and treatment activities (e.g., cancer screening, tobacco control, healthy eating, physical activity promotion) [33]. These agreements often offer some degree of flexibility in how states and local public health agencies use the funds. For example, state agencies can choose from a number of chronic disease prevention strategies provided by $\mathrm{CDC}$, e.g., improving access to healthy food and beverages, and what programs to implement to address a chosen strategy [34]. Often in larger grants, state health departments (SHD) serve as pass-through agencies to distribute funds, largely from DHHS, to contracted partner organizations, e.g., local 
health departments or community-based organizations. Local health departments, similar to local governments in Australia, provincial public health departments in Canada, and local authorities in the UK [35], are frontline organizations responsible for directly delivering public health programs to their towns or cities.

Within a SHD, chronic disease departments vary widely in terms of the level of hierarchy, specific positions and titles, and how governance is shared with local level agencies [36]. Regardless of the variation, decisionmaking is complex and shared among several types of practitioners: those in leadership positions (e.g., division directors, program managers) and those in lower-level positions (e.g., health educators). In SHDs, patterns and correlates of A-EBPs have been identified [31], and several studies have focused on awareness of, training for, and differences in A-EBPs in local public health settings $[23,25,32,37]$. Research about SHD practitioners' perspectives of the use of A-EBPs is limited, which is essential to support the development of strategies to support the use of A-EBPs that SHD practitioners would find relevant. Thus, the purpose of this study was to understand SHD practitioners' views about how A-EBPs are implemented and what hinders and supports their use.

\section{Methods}

This paper describes results from a qualitative study designed to understand the perspectives of SHD chronic disease practitioners about A-EBPs and how they relate to EBPH. The larger study from which this data comes was a cross-sectional study using a quantitative survey of SHD practitioners to examine capacity for EBPH in SHDs followed by a qualitative study using a subset of respondents from the quantitative survey.

\section{State site selection}

States were selected as part of a related evaluation of the National Association of Chronic Disease Directors' (NACDD) efforts to improve evidence-based public health capacity. NACDD is a professional organization dedicated to improving "the health of the public by strengthening state-based leadership and expertise for chronic disease prevention and control in states and at the national level" within the United States [38]. In 2015, a national survey was conducted of state chronic disease directors and state chronic disease prevention and health promotion program staff who were members of the $\mathrm{Na}$ tional Association of Chronic Disease Directors [31, 39]. States were ranked by overall capacity in EBPH using participants' responses to items in the national survey about the use of EBDM in their health department, public health accreditation status (accreditation indicating higher capacity), and the preventable burden of disease in each state. States were not eligible if they had fewer than five respondents to the national survey or were involved with the Prevention Research Center's STRIDE study [40]. Five respondents to the quantitative survey were needed for a state to be eligible because the study team deemed this enough response to represent the state's capacity for chronic disease EBPH and not just the capacity of individual practitioners. States involved in the STRIDE study were ineligible because this study focused on capacity building efforts for SHD practitioners, which would likely have influenced practitioners' reporting of related concepts to the same research team. From the ranking, a total of four state health departments were invited to participate in the study. In order to gain the perspectives of different types of state health departments, two states identified as higher capacity and two identified as lower capacity were included in the sample.

\section{Interview recruitment}

The Principal Investigator of the study emailed the Chronic Disease Director of each of the four states and invited the health department to participate in the study. Between five and eight state chronic disease prevention and health promotion program staff were recruited for the case study per state (four total states) using a snowball sampling technique. All chronic disease prevention or health promotion program staff, i.e., practitioners, in the state health departments of the four sampled states received an invitation to participate in an interview. Staff who were not involved in programmatic decisions or implementing programs were ineligible, e.g., data entry staff. Participants were told that the purpose of the study was to understand their opinions about evidence-based public health in their departments. Interviews occurred between December 2016 and May 2017. Participants who completed the interview were offered a $\$ 25$ donation to a non-profit organization from a list of options.

\section{Interview guide development}

Interview questions focused on gaining perceptions of evidence-based decision-making (Additional file 1). The research team and NACDD staff developed questions from the initial survey of SHD practitioners and previous research focused on evidence-based public health that was grounded in Diffusion of Innovations and Institutional Theory [40] and Aarons' conceptual model of evidence-based practice implementation in public service sectors $[41,42]$. The interview guide was divided them into four main sections: administrative support for evidence-based programs and policies, organizational support for evidence-based interventions, networks and partnerships to support evidence-based decision-making, and health equity. The focus of this paper is the first three sections, which included questions about access to 
and relevance of evidence, leaders' supports and expectations for EBDM, barriers and facilitators to implementing EBIs, types of partnerships, and recommendations for improving organizational supports for EBDM; the health equity data are presented elsewhere [43]. Training for interviewers included the purpose of the study, appropriate interview strategies, and questions on the interview guide. Thematic saturation was considered to be reached once no new categories of information or themes emerged from additional interviews, as decided by the research team [44]. For example, when research team discussions based on field notes indicated that the concepts of formal and informal communication of EBPH by leaders had been developed sufficiently and little new information on this concept was emerging in new interviews, that theme was considered saturated.

\section{Data analysis}

Interviews were analyzed according to qualitative research standards established by Hennink and colleagues [45]. Field notes were made after each call to supplement interpretation of interview coding. Each recorded interview was professionally transcribed. Transcripts were analyzed using NVivo (version 11) using the principles of thematic analysis, which emphasizes using a combination of deductive and inductive approaches to qualitative data analysis [46, 47]. A common codebook (Additional file 2) was developed deductively, i.e., codes that were developed from the interview guide, and inductively, i.e., codes were added as themes emerged from initial coding. Using a constant comparative coding technique [48], transcripts were coded using the initial codebook, and then recoded as additional themes emerged. Research assistants received one-on-one training from a staff member experienced in qualitative coding and were continually coached throughout the coding process. Fifty percent of the transcripts were coded by at least two people to increase consistency (three coders total for the project), and discrepancies were discussed with a third reviewer to come to a consensus on each inconsistency. Coded comments were synthesized into overall themes, and these themes were then further subdivided and categorized in NVivo (version 11). Results are presented by A-EBP domain, i.e., workforce development, leadership, organizational climate and culture, relationships and partnerships, and financial processes. There were no meaningful differences between high and low capacity states (the sampling approach), and the intent was not to examine differences by capacity. Thus, the results are presented overall and not stratified by state capacity. This study was approved by the Washington University in St. Louis Human Research Protection Office, and adherence to qualitative reporting guidelines is provided in Additional file 3.

\section{Results}

A total of 27 telephone interviews were completed to reach thematic saturation, with 5-8 interviews conducted with participants in each state. Interviews ranged from 21 to $77 \mathrm{~min}$ in length ( $\mathrm{mean}=60 \mathrm{~min}$ ). Chronic disease-related staff at state health departments had been in their current position for an average of $4.4 \pm 3.7$ years and had been working in public health for an average of $14.2 \pm 7.0$ years (Table 1 ).

\section{Workforce development}

Practitioners discussed two components related to workforce development: employees' training and experience upon hire and ongoing training opportunities.

Formal public health training and/or previous experience with evidence-based health practice were considered critical qualifications for a successful candidate that would be written into a job description formally. Additionally, an openness to learning and valuing EBDM were noted as important characteristics for potential employees that might be more tacit expectations not formally stated within a job description. Practitioners highlighted the importance of these qualities in light of staffing challenges in state and local public health departments, for example high turnover in many agencies.

Once employees are hired, ongoing professional development is delivered most commonly through trainings and webinars. Some participants noted a desire for trainings to help "program managers to think in a way that is ... to train them not only in the tools of an evidencebased approach, but also the way to think in terms of not necessarily accepting something because ... you've always done it, but to look at what the latest is, to promote an environment of active learning."

Variation in how much training opportunities are used depended on the internal capacity of the SHD and available funds to attend more expensive, external

Table 1 Sample characteristics $(n=27)$

Interviews per state

State 1

8

State 2

State 3

6

State 4

8

Number of staff supervised

Mean

SD

Number of people in unit

4.4

3.7

Years in current position

52.1

118.9

Years with organization

3.6

3.0

Years in public health

9.1

6.2

$S D$ standard deviation 
professional development opportunities like in-person conferences. Practitioners reported the availability of external training opportunities from organizations such as CDC and National Association of Chronic Disease Directors, but fewer opportunities internal to their own department. External opportunities for professional development were considered useful by the majority of practitioners and preferable over no opportunities; however, some practitioners expressed a desire to build capacity within their work units to develop internal trainings that can be tailored to the local context of the SHD, the chronic diseases of focus, and the specific professional development needs within the work unit.

\section{Leadership}

Leaders' commitment to evidence-based work was described as important to the overall functioning and mission of the SHD. One participant noted that "there's just a pretty high level of commitment to raising the performance of staff [through EBDM] and an understanding that it's essential to good public health practice." Participants described formal and informal ways that leadership communicates their expectations for EBPH processes. Formally, leaders institutionalized their expectations for EBPH through strategic plans.

The department has a strategic plan, and evidencebased public health is prominently displayed as an objective in the strategic plan. It's right up there with employee retention and reducing obesity as one of the main objectives of the department leadership.

Practitioners noted that by choosing to include language about the use of EBPH within these plans, leaders set the tone for the department and make opportunities for employees to use resources and training opportunities to improve their EBPH skills more available. Informal communication about EBPH in meetings, weekly emails, or other in-person interactions reinforced the formal expectations. In this regard, leaders implicitly communicated that there was "just [a] whole mindset change that this is what [the work unit was] going to be doing now. [They're] not going to do the same old same old, that you will make sure that you have evidence-base for what you're doing."

\section{Organizational climate and culture}

Participants noted that their organizational culture supported EBPH. An exemplary quote of this concept was "[the use of EBPH has] sort of reached a level of acceptance [within their work unit] that's so high that it's more remarkable for its absence." The expectation for using $\mathrm{EBPH}$ was sometimes discussed in tandem with the expectation of the $\mathrm{CDC}$ and/or leadership to use $\mathrm{EBPH}$, indicating that some practitioners viewed $\mathrm{EBPH}$ culture created in a top-down fashion. Some practitioners mentioned that the use of EBPH was limited by the lack of dedicated time for strategic planning and lack of evaluation data that is incorporated into strategic planning.

Practitioners expressed that being able to access evidence and use it in planning helps justify programmatic decisions to leaders. This information provides confidence and credibility in presenting information to stakeholders who might need to be convinced of priority issues. Additionally, access to knowledge about EBIs makes it easier for practitioners to “... know what [they're] going to measure... and to work with [their] local partners to be able to put that in place of, well here are [the] indicators and here's what [they should be] be measuring and supporting and why."

Practitioners reported a variety of available resources to access evidence in planning and implementing projects, including the CDC's Guide to Community Preventive Services (the Community Guide) and resources developed by NACDD and National Association of City and County Health Officials to summarize available EBIs. Primary literature was discussed as an important source of cutting-edge evidence; however, participants noted that digital access to literature is limited due to subscription fees and lack of knowledge on how to navigate digital libraries. Several mentioned digital library projects and resource banks and having student interns with access to journals through their educational institutes as ways of facilitating access to digital resources.

Many practitioners highlighted the importance of access to evidence that is relevant to the populations they serve. Practitioners considered the available evidence mostly relevant to their work and the populations they serve. When there is not a direct fit, they focus on balancing fidelity with fit when selecting and implementing EBIs. There was a suggestion that practitioners "can look at [an EBI] in terms of like this is the other state, so to apply that here in [our state], you always have to kind of tailor it for that context." Once an EBI has been tailored, practitioners noted that they can ensure fit within their community by working with their community members to pilot test materials and programs before implementation and with considering evidence in conjunction with local data. Participants often noted that evidence was not relevant for certain populations, such as different racial or ethnic groups or rural communities. Additionally, when practitioners are working within a pre-defined subset of the population, such as people with disabilities, there is sometimes too much variation within the subset of the population to know whether an EBI is applicable to the whole subset: 
When we're talking about sometime like ... chronic disease self-management programs for people with disabilities, and the disabilities definition is such a huge spectrum, that this evidence-based program might not be effective for some disability populations.

\section{Relationships and partnerships}

Partnerships were described as key to a SHD's ability to deliver EBIs to communities within their states, and the majority of partnerships described were with public sector organizations. Many of the partners were organizations in health-related sectors, such as medical providers, community-based organizations, and local public and tribal health agencies. However, several practitioners discussed the importance of partnerships in sectors not related to health, including religious organizations, higher education settings, and the state transportation department.

We kind of acknowledge that if we want to drive towards improved health outcomes we're not going to be able to do that ourselves. We'd have to look beyond our direct spheres of influence and try to partner with other sectors. Other sectors are actually going to have to potentially more of an impact on health outcomes than we can working alone in just our agency.

The connections were either developed organically, e.g., a partner organization and SHD are introduced based on mutual interest and create a project together, or by deliberately seeking out specific partners, e.g., a SHD reaches out to a community organization based on the particular needs of an existing project. Forming partnerships intentionally is facilitated by having a dedicated person who establishes and maintains collaborations. As one participant described,

... I just started to read a lot about the links between air quality and particulate air matter with cardiovascular disease and diabetes. So ... I just called over ... we have an air quality division that's in another building, like, can we meet? Can you tell me a little bit about where your hot spots are? And can we map those against where our hot spots are as far as heart failure? And there's got to be some ... a fair amount of overlap. And so I opened up a couple of opportunities for us to look at working particularly in those geographic areas.

Practitioners offered many recommendations for effectively establishing and maintaining partnerships. They should be thoughtfully developed with careful understanding of the audience and context of the partnering organization and community. Listening to and acknowledging the history of potential partners can help develop trusting and respectful relationships and frame discussion of the importance of EBPH.

... When you understand the history that helps us better frame the importance of these evidence-based processes, whereas if you're not hearing deeply and being thoughtful and understanding that community's history, it sounds like another government agency coming in and telling you how to live your life.

Additionally, buy-in and mutual agreement between partners and leadership was viewed as a supportive factor in implementation. Strategic planning should guide which partnerships are prioritized, as well as guide which partners have a shared goal or interest and can also benefit from the partnership. Negotiating what the common ground is may take time, humility, and patience.

... There may be health voluntary agencies that are very interested in addressing obesity. Their chosen approach has been ... [for schools to] ... conduct physical education. And that's been their issue. That may not necessarily be in concert with the education world around and they're trying to balance a number of things. As public health, we may be pushing like, yes, we like the idea of physical education. We can assess, based on political feasibility and readiness, but that's not where education wants to go. But what we can support is improving walkability to and from school to get those physical activity needs met.

Participants discussed that once a partnership is formed, it is important to be set expectations from the beginning, be reliable, and stay in regular communication with partners. However, communication should be intentional; respectful and efficient use of time is needed and having meetings just for the sake of having meetings may be detrimental.

Time, funding, and politics were identified as major barriers to forming and maintaining partnerships. The time it takes to build and maintain relationships can be too much for some departments to take on given competing interests within the department; in this case, quality over quantity may work better for some organizations. Some practitioners voiced that "... It is okay [to] have a small number of partnerships. What we need to achieve is the outcome, the policy, system and environmental change 
strategies. And if we can do that with five, then who cares that we didn't have 50 ?"

Also, forming partnerships may be challenging due to differing politics of potential partner organizations.

[In our state] there's a strong element of libertarianism, and even if something is evidence-based, ... it's shown to save lives, it's shown to be economical and saves the state money, you can always weaken support for it [with partners] saying, well that's just a nanny state.

Last, funding needs to be available to support partners' projects. Participants explained that this is often not the case, due to lack of flexible funding streams to support partner organizations. One participant noted that an existing partnership had to end because of a change in funds from those that would support personnel costs and direct program costs to funds that would only support direct program costs. As a result of this partnership ending, the program ended because there was no partner organization who could take on program implementation without financial support for personnel.

\section{Financial characteristics of the agency}

Most participants discussed funding, which the authors consider an agency-level characteristic, as it relates to EBI implementation. There was very little discussion of other aspects of finances, e.g., allocating resources for quality improvement. Funding was the top barrier to implementing EBIs that was mentioned by participants, and funding constraints were mentioned in relation to many of the other A-EBP domains. Funding was discussed in terms of the lack of total funding, restrictions on the way funding could be used, and bureaucracy related to funding. Practitioners described funding as a strong influence on the choice of EBI, noting "... funding pretty much drives everything. So if there's funding that's available and it's ... tied to a certain evidencebased strategy or program or intervention, ... you will follow that."

Practitioners expressed a desire for flexibility and creativity in gaining and using funding to address the specific needs of communities in their state. Additionally, participants noted they were sometimes frustrated with the way funding decisions are made in state legislatures. Instead of prioritizing funding based on a population health perspective, long-term funding sources are sometimes appropriated to address rare diseases based on vocal advocates such as when “... someone's appropriated a certain amount of funds forever on topic $\mathrm{X}$ because a mom whose kid had [a] condition got a bill passed that sets aside dollars forever related to that. And even if the condition, you know that you have one case a year in your whole state, and you really could better spend that money on something else, there's nothing you can do about it."

\section{Discussion}

This study gained important insight into state health department practitioners' perceptions of A-EBPs, which can influence the use of EBDM. Practitioners in all states reported that their work unit culture is supportive of EBPH processes and that leadership encouraged and expected staff to use evidence-based processes. Participants discussed the provision of trainings and technical assistance as key to workforce development and how leaders communicate their expectations. Access to evidence, partnerships, and funding restrictions were consistently discussed as barriers to the use of A-EBPs and EBDM. Findings from this study can be used to inform the development and refinement of efforts to improve the use of A-EBPs and organizational capacity of state health departments. Making changes at the organization level can be difficult, slow, and costly and involves buy-in from many stakeholders at multiple levels within organizations in complex public health systems [20, 32, 49, 50]. To this end, these interviews identified aspects of the organization that practitioners believe can make a difference to their work unit.

Many opportunities exist to increase access to evidence relevant for public health decision-making. Increasing access to evidence has been a cornerstone of efforts across the globe to improve EBDM, known as evidence-informed decision-making in settings outside of the United States. Organizations have been established to synthesize and translate evidence to public health decision makers in Canada [51], Australia [52], and the UK [13], and these organizations have developed and implemented training for accessing evidence (discussed in the next paragraph). Some practitioners use "grey" literature because peer-reviewed documents are behind journal paywalls [53]. To address this, the movement towards open access journals can facilitate the reach of peer-reviewed literature to practitioners, including open access fees that authors pay to freely distribute articles [54]. One promising strategy to increase access to evidence that is emerging in the Unites States is the academic health department (AHD) partnership, which is a formal or informal arrangement between an academic institution and a governmental public health agency providing mutual benefits in teaching, research, and practice [55-57]. Practitioners within AHD partnerships often have appointments within the academic setting and can therefore enhance their access to resources such as peer-reviewed articles using university credentials. Linkages with academics might allow practitioners to access information and library services provided by 
university librarians, which Peirson and colleagues noted as important along with accessing peer-reviewed literature [58]. Promoting and institutionalizing these partnerships as part of several Council for Education on Public Health accreditation standards for American schools and programs of public health would ensure broad and sustainable impact on the ability of practitioners to access evidence [59].

Training and leadership development are needed to build skills of the public health workforce $[60,61]$. Based on the results of this study, training topics could include information on accessing the literature and adapting EBIs so that they are relevant to the community. Previous and ongoing studies have demonstrated the benefit of different EBDM training approaches for individuals' confidence and skills related to EBDM [35, 62-67]. Within the United States, there are several training programs and materials available to public health practitioners, including those developed by the Regional Public Health Training Centers, the National Network of Public Health Institutes, the Public Health Foundation, CDC, universities, and professional groups such as the National Association of Chronic Disease Directors (e.g., STAR program). There is a reciprocal relationship between individuals, including leaders, and implementation climate [30,68-72]; thus, capacity for EBPH at the organizational level might be improved by increasing the capacity of those in leadership positions level in addition to the organizational level. Based on the findings in this study, key topics for leadership training include strategic planning, effective communication, and working with other leaders and lower level employees. Few trainings and interventions exist to directly change the organizational level factors (i.e., A-EBPs) [58, 62], which is likely important to implement in conjunction with individual-level trainings.

Strong leadership is crucial for successful EBPH, as leaders set the tone for organizational climate and culture [19, 20, 73-78]. In turn, employees interpret the culture as what management values and supports [79]. Leaders at multiple levels (e.g., deputy directors, division directors, program managers) play a specific role in promoting a culture of EBPH but need to work together to communicate a consistent message to employees and facilitate EBPH use $[79,80]$. As discussed in these interviews and in previous literature, there are many opportunities for leaders to facilitate the use of EBPH. Leaders can influence the implementation climate by role modeling and coaching effective use of EBPH [79]. Additionally, leaders can improve EBDM use communication of clear expectations for using EBPH, securing time and funds for employees to use EBDM, and including EBDM in strategic plans [58, 81]. The influence of a leader may differ by level; for example, Birken and colleagues identified specific roles of middle managers (e.g., program managers), including mediating between strategy and day-to-day operations [68].

Creating and maintaining partnerships was also described as key to successful EBDM and EBI implementation but an area that could be improved. This is especially important for state health departments or other agencies in a similar role, who rely on contracts with partner organizations to directly implement programs within a local community. In particular, forming non-traditional partnerships, including those with private sector organizations, holds promise to improve public health [82]. Regardless of the sector, static resources, e.g., The Community Tool Box's Creating and Maintaining Partnerships toolkit, exist to assist practitioners in strengthening and maintaining partnerships [83, 84]. Wider dissemination of these existing materials is needed to improve their reach and use. Resources such as interactive materials or trainings may help identify non-traditional partners, develop a common goal or mission, and create effective communication strategies with partners. Developing skills to interact with other stakeholders and organizations is nuanced and may require a more active approach than passive materials such as toolkits, for example through hands-on experience with practicebased research networks [85]. This should be integrated in practitioner training priorities.

The findings of this study are not without limitations. The relatively small sample in this study may limit its generalizability to other public health agencies in the US or globally. Also, the data gathered were the opinions of the staff members and may not represent the entire state health department. The responses from the staff members may have been subject to social desirability bias. Despite these limitations, these results are important for understanding what practitioners themselves think of the role of A-EBPs in promoting EBPH and their needs to improve capacity.

\section{Conclusions}

Future studies and practice-based work is needed to understand the best ways to translate this information into programs, practices, and policies that can improve public health departments' use of EBPH. As discussed in these interviews and found in previous literature, an approach for improving public health capacity that is tailored to the specific organizational structure and context is likely to be most effective [30]. Additionally, modifications to funding, e.g., reducing restrictions on how funding can be used to implement evidence-based interventions, are needed to allow practitioners to effectively implement programs that can improve the public's health and wellbeing. 


\section{Supplementary information}

Supplementary information accompanies this paper at https://doi.org/10. 1186/s43058-020-00003-x.
Additional file 1. Interview guide
Additional file 2. Coding Tree
Additional file 3. Reporting Checklist

\section{Abbreviations}

A-EBP: Administrative evidence-based practices; CDC: Centers for Disease Control and Prevention; EBDM: Evidence-based decision-making; EBI: Evidence-based intervention; EBPH: Evidence-based public health; NACDD: National Association of Chronic Disease Directors; SHD: State health department

\section{Acknowledgements}

We appreciate the SHD practitioners for their participation in our interviews. With gratitude, we acknowledge the administrative support of Linda Dix and Mary Adams at the Prevention Research Center in St. Louis, Brown School, Washington University in St. Louis.

\section{Authors' contributions}

$A A E$ and RCB contributed to the conceptualization and design. CAV, AAE and RCB contributed to the interview guide development. CAV and AL contributed to the interview coding and summary. SM, CAV, AAE, MM, AL, $J A$, JR, and RCB contributed to the review of the findings. SM, CAV, and RCB contributed to the writing. SM, CAV, AAE, MM, AL, JA, JR, and RCB contributed to the manuscript content revisions. All authors read and approved the final manuscript.

\section{Funding}

This study was supported, in part, by the National Association of Chronic Disease Directors agreement number 1612017. The funding body assisted in reviewing and developing the interview guide and contributed to the review and interpretation of findings.

\section{Availability of data and materials}

Please contact the lead author for more information.

\section{Ethics approval and consent to participate}

All participants completed the study consent form. Study procedures and materials including the consent form were submitted by the second author and approved by the Washington University in St. Louis Institutional Review Board on July 22, 2016. All study staff have been trained in ethical conduct of human subjects research.

\section{Consent for publication}

Not applicable.

\section{Competing interests}

The authors declare that they have no competing interests.

\section{Author details}

${ }^{1}$ Prevention Research Center in St. Louis, Brown School, Washington University in St. Louis, One Brookings Drive, Campus Box 1196, St. Louis, MO 63130, USA. ${ }^{2}$ National Association of Chronic Disease Directors, Decatur, GA, USA. ${ }^{3}$ Arcora Foundation, Seattle, WA, USA. ${ }^{4}$ Department of Surgery (Division of Public Health Sciences) and Alvin J. Siteman Cancer Center, Washington University School of Medicine, Washington University in St. Louis, St. Louis, $\mathrm{MO}, \mathrm{USA}$.

Received: 13 August 2019 Accepted: 8 January 2020 Published online: 25 February 2020

\section{References}

1. World Health Organization. Noncommunicable diseases country profiles, vol. 2018; 2018. Available from: https:/www.who.int/nmh/countries/2018/usa_en.pdf?ua=1

2. Buttorff C, Ruder T, Bauman M. Multiple chronic conditions in the United States. Rand Corp: Santa Monica; 2017.
3. Center for Medicare \& Medicaid Services. National Health Expenditures 2017 Highlights. 2018.

4. Elders MJ, Perry CL, Eriksen MP, Giovino GA. The report of the surgeon general: preventing tobacco use among young people. Am J Public Health. 1994;84(4):543-7.

5. Eyre $H$, Kahn R, Robertson RM, American Cancer Society tADA, the American Heart Association. Collaborative Writing C. Preventing cancer, cardiovascular disease, and diabetes: a common agenda for the American Cancer Society, the American Diabetes Association, and the American Heart Association. Diabetes Care. 2004;27(7):1812-24.

6. Lorig KR, Ritter P, Stewart AL, Sobel DS, Brown BW Jr, Bandura A, et al. Chronic disease self-management program: 2-year health status and health care utilization outcomes. Med Care. 2001:39(11):1217-23.

7. U.S. Department of Health and Human Services. Physical activity guidelines for Americans. 2nd ed. Washington, D.C: U.S. Department of Health and Human Services; 2018

8. World Health Organization. Diet, nutrition and the prevention of chronic diseases. World Health Organ Tech Rep Ser. 2003;916:i.

9. Warnecke RB, Oh A, Breen N, Gehlert S, Paskett E, Tucker KL, et al. Approaching health disparities from a population perspective: the National Institutes of Health Centers for Population Health and Health Disparities. Am J Public Health. 2008;98(9):1608-15.

10. American Public Health Association. Supporting research and evidencebased public health practice in state and local health agencies. 2017. Available from: https://www.apha.org/policies-and-advocacy/public-healthpolicy-statements/policy-database/2018/01/18/supporting-research-andevidence-based-public-health-practice.

11. Canadian Institutes of Health Research. Canadian Institutes of Health Research Act. 2019 Available from: https://laws.justice.gc.ca/PDF/C-18.1.pdf.

12. National Health and Medical Research Council. Research translation [Available from: https://www.nhmrc.gov.au/research-policy/researchtranslation-and-impact. Accessed 11 Dec 2019.

13. National Institute for Health and Care Excellence. What we do [Available from: https://www.nice.org.uk/about/what-we-do. Accessed 11 Dec 2019.

14. Brownson RC, Baker EA, Deshpande AD, Gillespie KN. Evidence-based public health. New York: Oxford university press; 2017.

15. Brownson RC, Gurney JG, Land GH. Evidence-based decision making in public health. J Public Health Manag Pract. 1999;5(5):86-97.

16. Macintyre S, Petticrew M. Good intentions and received wisdom are not enough. J Epidemiol Community Health. 2000;54(11):802-3.

17. Tannahill A. Beyond evidence--to ethics: a decision-making framework for health promotion, public health and health improvement. Health Promot Int. 2008:23(4):380-90.

18. Brownson RC, Allen P, Jacob RR, deRuyter A, Lakshman M, Reis RS, et al. Controlling chronic diseases through evidence-based decision making: a group-randomized trial. Prev Chronic Dis. 2017;14:E121.

19. Dodson EA, Baker EA, Brownson RC. Use of evidence-based interventions in state health departments: a qualitative assessment of barriers and solutions. J Public Health Manag Pract. 2010;16(6):E9-E15.

20. Jacobs JA, Dodson EA, Baker EA, Deshpande AD, Brownson RC. Barriers to evidence-based decision making in public health: a national survey of chronic disease practitioners. Public Health Rep. 2010;125(5):736-42.

21. Jacobs JA, Jones E, Gabella BA, Spring B, Brownson RC. Tools for implementing an evidence-based approach in public health practice. Prev Chronic Dis. 2012:9:E116.

22. Maylahn C, Fleming D, Birkhead G. Health departments in a brave new world. Prev Chronic Dis. 2013;10:E41.

23. Brownson RC, Allen P, Duggan K, Stamatakis KA, Erwin PC. Fostering moreeffective public health by identifying administrative evidence-based practices: a review of the literature. Am J Prev Med. 2012:43(3):309-19.

24. Brownson RC, Baker EA, Left TL, Gillespie KN, True WR. Evidence-based public health; 2011. p. 1-312

25. Brownson RC, Fielding JE, Maylahn CM. Evidence-based public health: a fundamental concept for public health practice. Annual review of public health; 2009. p. 175-201

26. Fielding JE, Briss PA. Promoting evidence-based public health policy: can we have better evidence and more action? Health Aff. 2006;25(4):969-78.

27. Glasziou P, Longbottom H. Evidence-based public health practice. Aust N Z J Public Health. 1999;23(4):436-40.

28. Jenicek M. Epidemiology, evidenced-based medicine, and evidence-based public health. J Epidemiol. 1997;7(4):187-97. 
29. Public Health Accreditation Board. Public Health Accreditation Board Standards and Measures, version 1.5. Alexandria: Public Health Accreditation Board; 2013. Available from: http://www.phaboard.org/wp-content/uploads/ SM-Version-1.5-Board-adopted-FINAL-01-24-2014.docx.pdf

30. Brownson RC, Fielding JE, Green LW. Building capacity for evidence-based public health: reconciling the pulls of practice and the push of research. Annu Rev Public Health. 2018;39:27-53.

31. Eyler AA, Valko C, Ramadas R, Macchi M, Fershteyn Z, Brownson RC. Administrative evidence-based practices in state chronic disease practitioners. Am J Prev Med. 2018;54(2):275-83.

32. Brownson RC, Reis RS, Allen P, Duggan K, Fields R, Stamatakis KA, et al. Understanding administrative evidence-based practices: findings from a survey of local health department leaders. Am J Prev Med. 2014;46(1):49-57.

33. Public Law 95-224: Federal Grant and Cooperative Agreement Act of 1977. (92 Stat. 3; Date: February 3, 1978). Text from: United States Public Laws. Available from: Nexis Uni. Accessed 3 Dec 2019.

34. Rutledge GE, Lane K, Merlo C, Elmi J. Coordinated approaches to strengthen state and local public health actions to prevent obesity, diabetes, and heart disease and stroke. Prev Chronic Dis. 2018;15(14):170493.

35. Armstrong R, Waters E, Moore L, Dobbins M, Pettman T, Burns C, et al. Understanding evidence: a statewide survey to explore evidence-informed public health decision-making in a local government setting. Implement Sci. 2014;9:188.

36. Centers for Disease Control and Prevention. Health department governance 2018 Available from: https://www.cdc.gov/publichealthgateway/ sitesgovernance/index.html.

37. Erwin PC, Harris JK, Smith C, Leep CJ, Duggan K, Brownson RC. Evidencebased public health practice among program managers in local public health departments. J Public Health Manag Pract. 2014;20(5):472-80.

38. National Association of Chronic Disease Directors. About NACDD [Available from: www.chronicdisease.org/page/About_NACDD. Accessed 9 Dec 2019.

39. Furtado KS, Brownson C, Fershteyn Z, Macchi M, Eyler A, Valko C, et al. Health departments with a commitment to health equity: a more skilled workforce and higher-quality collaborations. Health Aff. 2018;37(1):38-46.

40. Allen P, Sequeira S, Jacob RR, Hino AAF, Stamatakis KA, Harris JK, et al. Promoting state health department evidence-based cancer and chronic disease prevention: a multi-phase dissemination study with a cluster randomized trial component. Implement Sci. 2013;8(1):141.

41. Aarons GA, Hurlburt M, Horwitz SM. Advancing a conceptual model of evidence-based practice implementation in public service sectors. Adm Policy Ment Health Ment Health Serv Res. 2011;38(1):4-23.

42. Jacobs JA, Duggan K, Erwin P, Smith C, Borawski E, Compton J, et al. Capacity building for evidence-based decision making in local health departments: scaling up an effective training approach. Implement Sci. 2014;9(1):124.

43. Eyler AA, Valko CA, Macchi M, Fershteyn Z, Mazzucca SL, Brownson CA, et al. Adjusting the equity lens: gaps in addressing health equity in state chronic disease prevention. Health Equity. 2019;3(1):86-91.

44. Corbin J, Strauss A. Theoretical sampling. Basics of qualitative research. 4th ed. Thousand Oaks: Sage Publications; 1990. p. 139-40.

45. Hennink MM, Hutter I, Bailey A. Qualitative research methods. London, UK. Thousand Oaks: SAGE Publications; 2011.

46. Braun V, Clarke V. Using thematic analysis in psychology. Qual Res Psychol. 2006;3(2):77-101

47. Fereday J, Muir-Cochrane E. Demonstrating rigor using thematic analysis: a hybrid approach of inductive and deductive coding and theme development. Int J Qual Methods. 2006;5(1):80-92.

48. Glazer B. The constant comparative method of qualitative analysis Grounded Theory Rev. 2008;7(3):436-45.

49. Ciliska D, Hayward S, Dobbins M, Brunton G, Underwood J. Transferring public-health nursing research to health-system planning: assessing the relevance and accessibility of systematic reviews. Can J Nurs Res. 1999; 31(1):23-36

50. Kneale D, Rojas-García A, Raine R, Thomas J. The use of evidence in English local public health decision-making: a systematic scoping review. Implement Sci. 2017;12(1):53.

51. Public Health Agency of Canada. National Collaborating Centres for Public Health 2017 Available from: https://www.canada.ca/en/public-health/services/ public-health-practice/national-collaborating-centres-public-health.html.

52. Cochrane Public Health. About Cochrane Public Health (CPH) [Available from: https://ph.cochrane.org/about-cochrane-public-health-cph. Accessed 11 Dec 2019.
53. Martin W, Wharf Higgins J, Pauly BB, MacDonald M. "Layers of translation" evidence literacy in public health practice: a qualitative secondary analysis. BMC Public Health. 2017;17(1):803.

54. Brownson RC, Eyler AA, Harris JK, Moore JB, Tabak RG. Getting the word out: new approaches for disseminating public health science. J Public Health Manag Pract. 2018;24(2):102-11.

55. Erwin PC, Keck CW. The academic health department: the process of maturation. J Public Health Manag Pract. 2014;20(3):270-7.

56. Erwin PC, Parks RG, Mazzucca S, Allen P, Baker EA, Hu H, et al. Evidencebased public health provided through local health departments: importance of academic-practice partnerships. Am J Public Health. 2019; 109(5):739-47.

57. The Council on Linkages Between Academia and Public Health Practice. Academic health departments: core concepts 2011 Available from: http:// www.phf.org/resourcestools/Documents/AHD_Concepts_2011Jan14.pdf.

58. Peirson L, Ciliska D, Dobbins M, Mowat D. Building capacity for evidence informed decision making in public health: a case study of organizational change. BMC Public Health. 2012;12:137.

59. Council on Education for Public Health. Accreditation criteria: schools of public health \& public health programs silver spring, MD. 2016 Available from: https://media.ceph.org/wp_assets/2016.Criteria.pdf.

60. Allen P, Jacob RR, Lakshman M, Best LA, Bass K, Brownson RC. Lessons learned in promoting evidence-based public health: perspectives from managers in state public health departments. J Community Health. 2018; 43(5):856-63.

61. Baker EL. Addressing urgent public health workforce needs: building informatics competency and strengthening management and leadership skills. J Public Health Manag Pract. 2015;21(Suppl 6):S5-6.

62. Armstrong R, Waters E, Dobbins M, Anderson L, Moore L, Petticrew M, et al. Knowledge translation strategies to improve the use of evidence in public health decision making in local government: intervention design and implementation plan. Implement Sci. 2013:8:121.

63. Serrano N, Diem G, Grabauskas V, Shatchkute A, Stachenko S, Deshpande A, et al. Building the capacity - examining the impact of evidence-based public health trainings in Europe: a mixed methods approach. Glob Health Promot. 2019:1757975918811102.

64. Waters E, Armstrong R, Swinburn B, Moore L, Dobbins M, Anderson L, et al. An exploratory cluster randomised controlled trial of knowledge translation strategies to support evidence-informed decision-making in local governments (The KT4LG study). BMC Public Health. 2011;11:34.

65. Pettman TL, Armstrong R, Jones K, Waters E, Doyle J. Cochrane update: building capacity in evidence-informed decision-making to improve public health. J Public Health. 2013;35(4):624-7.

66. Yousefi Nooraie R, Marin A, Hanneman R, Lohfeld L, Dobbins M. Implementation of evidence-informed practice through central network actors; a case study of three public health units in Canada. BMC Health Serv Res. 2017;17(1):208.

67. Diem G, Brownson RC, Grabauskas V, Shatchkute A, Stachenko S. Prevention and control of noncommunicable diseases through evidence-based public health: implementing the NCD 2020 action plan. Glob Health Promot. 2016; 23(3):5-13.

68. Birken S, Clary A, Tabriz AA, Turner K, Meza R, Zizzi A, et al. Middle managers' role in implementing evidence-based practices in healthcare: a systematic review. Implement Sci. 2018;13(1):149.

69. Kislov $\mathrm{R}$, Waterman $\mathrm{H}$, Harvey $\mathrm{G}$, Boaden R. Rethinking capacity building for knowledge mobilisation: developing multilevel capabilities in healthcare organisations. Implement Sci. 2014;9:166.

70. Meyer AM, Davis M, Mays GP. Defining organizational capacity for public health services and systems research. J Public Health Manag Pract. 2012; 18(6):535-44.

71. Robinson K, Farmer T, Riley B, Elliott SJ, Eyles J, CHHDP Investigative Team S, et al. Realistic expectations: investing in organizational capacity building for chronic disease prevention. Am J Health Promot 2007;21(5):430-438.

72. Schneider B, Ehrhart MG, MacEy WH. Organizational climate and culture Ann Rev Psychol. 2013;64:361-88.

73. Oliver K, Innvar S, Lorenc T, Woodman J, Thomas J. A systematic review of barriers to and facilitators of the use of evidence by policymakers. BMC Health Serv Res. 2014;14:2

74. Orton L, Lloyd-Williams F, Taylor-Robinson D, O'Flaherty M, Capewell S. The use of research evidence in public health decision making processes: systematic review. PLoS One. 2011;6(7):e21704. 
75. van de Goor I, Hämäläinen R-M, Syed A, Juel Lau C, Sandu P, Spitters H, et al. Determinants of evidence use in public health policy making: results from a study across six EU countries. Health Policy. 2017;121(3):273-81.

76. Zardo P, Collie A. Predicting research use in a public health policy environment: results of a logistic regression analysis. Implement Sci. 2014;9:142.

77. Zardo P, Collie A. Type, frequency and purpose of information used to inform public health policy and program decision-making. BMC Public Health. 2015;15:381.

78. Zardo P, Collie A, Livingstone C. Organisational factors affecting policy and programme decision making in a public health policy environment. Evid Policy. 2015;11(4):509-27.

79. Aarons GA, Ehrhart MG, Farahnak LR, Sklar M. Aligning leadership across systems and organizations to develop a strategic climate for evidencebased practice implementation. Annu Rev Public Health. 2014;35:255-74.

80. Chreim S, Williams BE, Coller KE. Radical change in healthcare organization mapping transition between templates, enabling factors, and implementation processes. J Health Organ Manag. 2012;26(2):215-36.

81. Dobbins M, Traynor RL, Workentine S, Yousefi-Nooraie R, Yost J. Impact of an organization-wide knowledge translation strategy to support evidenceinformed public health decision making. BMC Public Health. 2018;18(1):1412.

82. Simon PA, Fielding JE. Public health and business: a partnership that makes cents. Health Aff. 2006;25(4):1029-39.

83. National Association of Chronic Disease Directors. Partnership Analysis and Enhancement Toolkit Resources [Available from: http://nacddarchive.org/ nacdd-initiatives/cardiovascular-health/professional-development/ Partnership/partnership-assessment-resources. Accessed 6 May 2019

84. The Community Tool Box. Creating and maintaining partnerships: center for community health and development; 2018 Available from: https://ctb.ku. edu/en/creating-and-maintaining-partnerships.

85. Mays GP, Hogg RA, Castellanos-Cruz DM, Hoover AG, Fowler LC. Public health research implementation and translation: evidence from practicebased research networks. Am J Prev Med. 2013;45(6):752-62.

\section{Publisher's Note}

Springer Nature remains neutral with regard to jurisdictional claims in published maps and institutional affiliations.

Ready to submit your research? Choose BMC and benefit from:

- fast, convenient online submission

- thorough peer review by experienced researchers in your field

- rapid publication on acceptance

- support for research data, including large and complex data types

- gold Open Access which fosters wider collaboration and increased citations

- maximum visibility for your research: over $100 \mathrm{M}$ website views per year

At $\mathrm{BMC}$, research is always in progress.

Learn more biomedcentral.com/submissions 